\title{
Massive chest wall resection and reconstruction for malignant disease
}

This article was published in the following Dove Press journal:

OncoTargets and Therapy

19 April 2016

Number of times this article has been viewed

\author{
Christophoros N Foroulis' \\ Athanassios D Kleontas' \\ George Tagarakis' \\ Chryssoula Nana' \\ loannis Alexiou' \\ Vasilis Grosomanidis' \\ Paschalis Tossios' \\ Elena Papadaki \\ loannis Kioumis ${ }^{2}$ \\ Sofia Baka ${ }^{3}$ \\ Paul Zarogoulidis ${ }^{2}$ \\ Kyriakos Anastasiadis \\ 'Department of Cardiothoracic \\ Surgery, Aristotle University School of \\ Medicine, AHEPA University Hospital, \\ 2Pulmonary Department-Oncology \\ Unit, "G. Papanikolaou” General \\ Hospital, Aristotle University of \\ Thessaloniki, ${ }^{3}$ Oncology Department, \\ European Interbalkan Medical Center, \\ Thessaloniki, Greece
}

Correspondence: Paul Zarogoulidis Pulmonary Department-Oncology Unit, "G. Papanikolaou” General Hospital, Aristotle University of Thessaloniki, Exohi I 100, Thessaloniki 57010, Greece

Tel +30 697727 I974

Fax +3023I0 992424

Email pzarog@hotmail.com
Objective: Malignant chest wall tumors are rare neoplasms. Resection with wide-free margins is an important prognostic factor, and massive chest wall resection and reconstruction are often necessary. A recent case series of 20 consecutive patients is reported in order to find any possible correlation between tumor histology, extent of resection, type of reconstruction, and adjuvant treatment with short- and long-term outcomes.

Methods: Twenty patients were submitted to chest wall resection and reconstruction for malignant chest wall neoplasms between 2006 and 2014. The mean age (ten males) was 59 \pm 4 years. The size and histology of the tumor, the technique of reconstruction, and the short- and longterm follow-up records were noted.

Results: The median maximum diameter of tumors was $10 \mathrm{~cm}(5.4-32 \mathrm{~cm})$. Subtotal sternal resection was performed in nine cases, and the resection of multiple ribs was performed in eleven cases. The median area of chest wall defect was $108 \mathrm{~cm}^{2}\left(60-340 \mathrm{~cm}^{2}\right)$. Histology revealed soft tissue, bone, and cartilage sarcomas in 16 cases (80\%), most of them chondrosarcomas. The rest of the tumors was metastatic tumors in two cases and localized malignant pleural mesothelioma and non-Hodgkin lymphoma in one case. The chest wall defect was reconstructed by using the "sandwich technique" (propylene mesh/methyl methacrylate/propylene mesh) in nine cases of large anterior defects or by using a $2 \mathrm{~mm}$ polytetrafluoroethylene (e-PTFE) mesh in nine cases of lateral or posterior defects. Support from a plastic surgeon was necessary to cover the fullthickness chest wall defects in seven cases. Adjuvant oncologic treatment was administered in 13 patients. Local recurrences were observed in five cases where surgical reintervention was finally necessary in two cases. Recurrences were associated with larger tumors, histology of malignant fibrous histiocytoma, and initial incomplete resection or misdiagnosis made by nonthoracic surgeons. Three patients died during the study period because of recurrent disease or complications of treatment for recurrent disease.

Conclusion: Chest wall tumors are in their majority mesenchymal neoplasms, which often require major chest wall resection for their eradication. Long-term survival is expected in lowgrade tumors where a radical resection is achieved, while big tumors and histology of malignant fibrous histiocytoma are connected with the increase rate of recurrence.

Keywords: chest wall tumors, chest wall resection, chest wall reconstruction, soft tissue sarcomas, sternal tumors, chondrosarcoma

\section{Introduction}

Chest wall tumors are defined as primary or metastatic to the chest wall tumors or tumors of the neighboring organs invading the contiguity of the chest wall. Primary malignant chest wall tumors are in their majority mesenchymal neoplasms and arise from the soft tissues, bones, and cartilages of the chest wall. ${ }^{1-6}$ Chest wall tumors are rare neoplasms and account for $<5 \%$ of all thoracic malignancies. ${ }^{3,6}$ Radical resection with wide tumor-free specimen margins is considered to be the most important prognostic factor for prolonged 
survival. Massive chest wall resection is often necessary to achieve a tumor-free margin resection, while reconstruction of both the skeletal and soft tissue defects is very important for the overall outcome. ${ }^{1-6}$

A recent case series of 20 consecutive patients who underwent curative massive chest wall resection and reconstruction for malignant chest wall tumors is reported in order to find any possible correlation between tumor histology, extent of resection, type of reconstruction, and adjuvant treatment with outcomes and quality of life.

\section{Patients and methods Patients}

The institutional review board of AHEPA University Hospital approved this study. Written informed consent was obtained from all patients. Twenty consecutive patients who underwent massive chest wall resection and reconstruction for malignant chest wall tumors from January 2006 to December 2014 (8 years) were included in the retrospective analysis. Massive chest wall resection was defined as the resection of $>4$ consecutive ribs or any subtotal/total sternal resection requiring reconstruction to maintain the stability of chest wall and to avoid paradoxical chest wall motion. Patients with primary non-small-cell lung cancer invading the chest wall were not included in the study. The mean age of 20 patients included in the study patients was $59 \pm 4$ years (variance: 15-80 years) with equal distribution between the two sexes (ten males and ten females).

\section{Preoperative workup}

Core needle biopsy was the only approach to obtain a tissue biopsy before curative surgery of a chest wall tumor if the radiographic diagnosis was evident. The results of core needle biopsy were inconclusive in one patient with non-Hodgkin lymphoma of the chest wall who underwent curative resection of the mass. By principle, incisional biopsies were not performed to avoid the spilling of soft tissues by neoplastic cells. Two patients had a preoperative diagnosis coming from incomplete tumor resections performed by nonthoracic surgeons. In another case, misdiagnosis as chest wall abscess was made by a nonthoracic surgeon, where the establishment of the final diagnosis of malignant fibrous histiocytoma (MFH) was made with considerable delay.

Magnetic resonance imaging of the chest was applied in selected patients to delineate possible invasion of adjacent structures, such as the thoracic outlet, the spine, and the mediastinal vessels. One patient with sternal chondrosarcoma underwent preoperative positron emission tomography/computed tomography (PET/CT) scan where

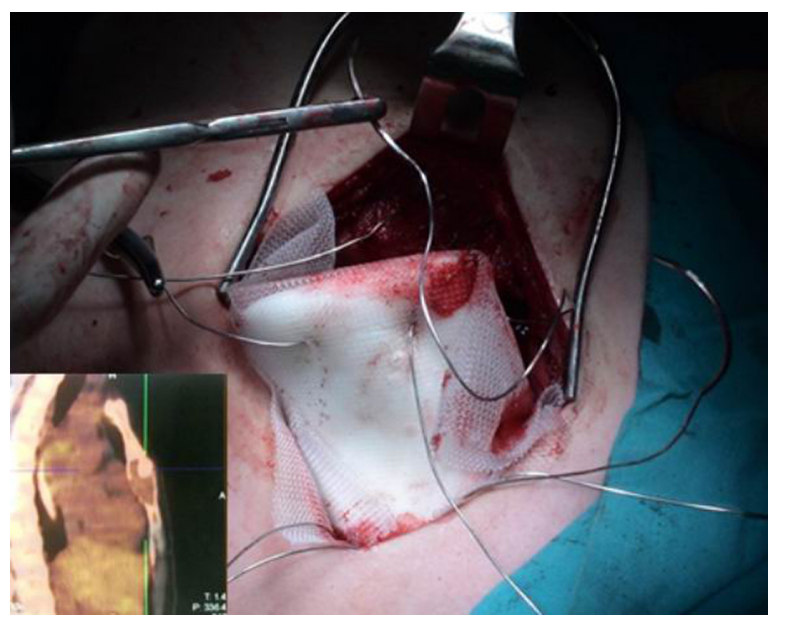

Figure I Sandwiched methyl methacrylate prosthesis for sternal reconstruction after the resection of sternal chondrosarcoma.

Notes: Four stainless steel wires fix the prosthesis to the remnants of the sternum to avoid dislocation. Inset: PET/CT scan showing the sternal tumor: low FDG uptake by the tumor (SUV $\left.V_{\max }=1.7\right)$.

Abbreviations: $\mathrm{PET} / \mathrm{CT}$, positron emission tomography/computed tomography; FDG, Fluorodeoxyglucose (I8F); SUV, standardized uptake value.

the Fluorodeoxyglucose (18F) (FDG) uptake by the tumor was considerably low as it was detected by the low SUVof 1.7 (Figure 1, inset). All patients had preoperative pulmonary function tests, while thorough preoperative assessment of the cardiac function was individually made. The decision to proceed with surgery was always made within a multidisciplinary oncology team. Plastic surgeons were involved in the preoperative planning when a fullthickness chest wall resection had to be made for radical tumor resection.

\section{Histology of the included patients in the study tumors}

The majority of the patients included in this study had primary chest wall sarcomas (16/20 cases $-80 \%)$, most of them are chondrosarcomas. Two patients had metastatic chest wall tumors where the preoperative workup did not reveal any other metastasis elsewhere in the body, while the primary site was well controlled. More details on tumor histologies are presented in Table 1.

\section{Chest wall reconstruction}

Reconstruction of the bony chest wall defect was made either by using the sandwich technique or by applying a dual-sided $2 \mathrm{~mm}$ e-PTFE mesh. The sandwich technique consisted of a layer of $1 \mathrm{~cm}$ of synthetic bony cement (methyl methacrylate), which was encountered within two layers of polypropylene mesh to form a rigid construction. The whole construction could be easily tailored to the size and convex of the bony defect during hardening of the synthetic cement. 
Table I Histology of the resected tumors

\begin{tabular}{ll}
\hline Histologic type & $\begin{array}{l}\text { Number } \\
\text { of patients }\end{array}$ \\
\hline Chondrosarcoma & 7 \\
Malignant fibrous histiocytoma & 3 \\
Malignant solitary fibrous tumor & 2 \\
Malignant peripheral nerve sheath tumor & $\mathrm{I}$ \\
Rhabdomyosarcoma & $\mathrm{I}$ \\
PNET & $\mathrm{I}$ \\
Fibrosarcoma & $\mathrm{I}$ \\
Metastatic oral squamous cell carcinoma to the sternum & $\mathrm{I}$ \\
Metastatic to the sternum thyroid carcinoma & $\mathrm{I}$ \\
Localized malignant mesothelioma & $\mathrm{I}$ \\
Primary chest wall non-Hodgkin lymphoma (DLBCL) & $\mathrm{I}$ \\
Total & 20 \\
\hline
\end{tabular}

Abbreviations: DLBCL, diffuse large B-cell lymphoma; PNET, primary chest wal Ewing's sarcoma.

The prosthesis was then fixed by propylene sutures to the surrounding soft tissues. Four stainless steel sutures passing through each corner of the prosthesis through the cement were applied to fix firmly the prosthesis to the surrounding bony skeleton, ie, to the ribs or to the remnant of sternum or clavicles, in order to avoid dislocation of the prosthesis (Figure 1). Regarding the dual-sided $2 \mathrm{~mm}$ e-PTFE mesh, it is a strong mesh that can be tailored to the size of any bony defect. The mesh was fixed to the edges of the chest wall defect with polypropylene sutures (Figures 2 and 3), trying always to tighten as much as possible the mesh, so as to resemble like a drum membrane.

The choice of the material used to cover the chest wall defect was mainly based on the location of the defect. Laterally or posteriorly located defects were reconstructed by the e-PTFE mesh (eleven cases), while anterior defects, especially those including part of or the whole sternum, were reconstructed by the sandwich technique (nine cases).

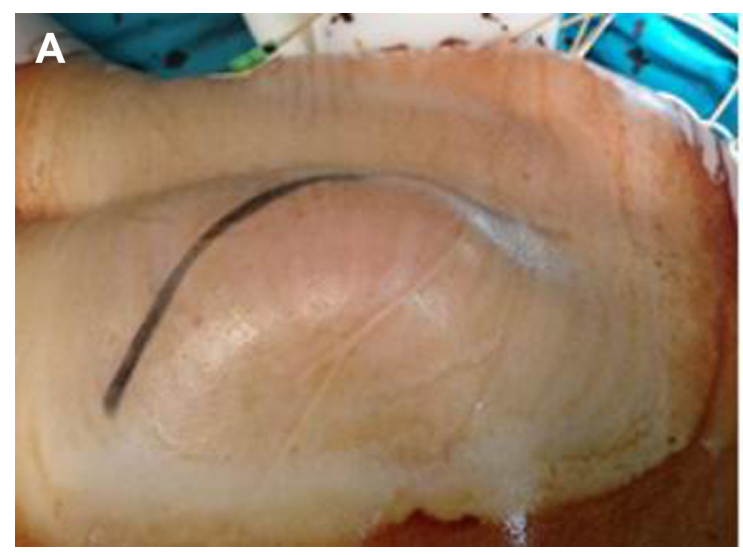

\section{Short- and long-term outcomes}

Short-term outcomes were defined as the mortality and morbidity of the procedures and any incomplete resection requiring redo surgery to achieve a final $\mathrm{R} 0$ oncologic result. Long-term outcomes were defined as any tumor recurrence, local or distant, and the long-term survival. Local recurrence was defined as the development of tumor within or adjacent to the chest wall resection/reconstruction area.

\section{Statistical analysis}

Due to the small number of cases as well as the heterogeneity of the tumors included in the study, the results are presented with a descriptive manner, since the data are not suitable for statistical analysis, with the exception of some descriptive statistics.

\section{Results}

The maximal diameter of chest wall masses varied between $5.4 \mathrm{~cm}$ and $32 \mathrm{~cm}$ (median: $10 \mathrm{~cm}$ ). The area of the bony chest wall defect resulting after tumor resection varied from $60 \mathrm{~cm}^{2}$ to $340 \mathrm{~cm}^{2}$ (median: $108 \mathrm{~cm}^{2}$ ). The area of the chest wall defect was measured to be $<100 \mathrm{~cm}^{2}$ in eight patients, $100-200 \mathrm{~cm}^{2}$ in seven patients, $200-300 \mathrm{~cm}^{2}$ in four patients, and $>300 \mathrm{~cm}^{2}$ in one patient. The maximum size of tumor and the area of chest wall defect were obtained from the final pathology reports.

\section{Tumor resection and reconstruction techniques}

The type and extent of resection and the applied chest wall reconstruction technique for particular cases are shown in Table 2.

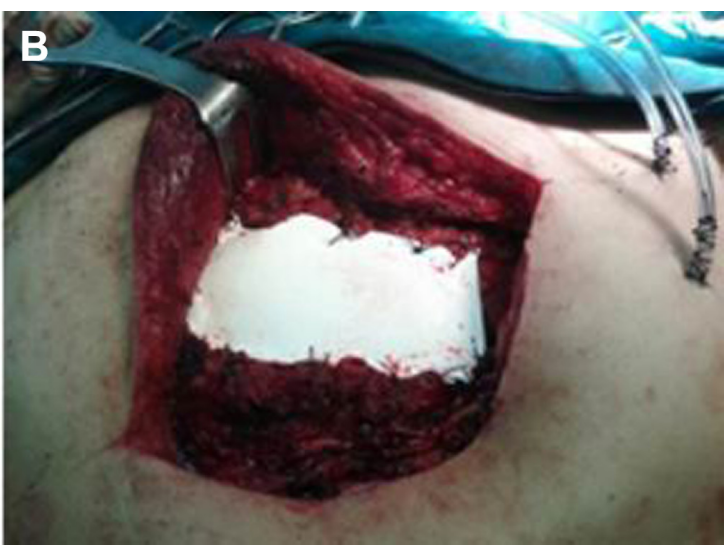

Figure 2 surgery specimens.

Notes: (A) Large malignant SFT of the posterior chest wall. (B) Reconstruction of the chest wall defect with 2 mm e-PTFE mesh after resection of the SFT. Abbreviations: SFT, solitary fibrous tumor; e-PTFE, polytetrafluoroethylene. 
Table 2 Reconstruction techniques according to the type and extent of chest wall tumor resection

\section{Cases}

Reconstruction with the sandwich technique

Subtotal sternal resection including the attached costal cartilages

Subtotal sternal resection en bloc with the proximal segments of both clavicles, the thymus and part of anterior pericardium

Subtotal sternal resection en bloc with the thymus gland, anterior pericardium, adventitia of the ascending aorta, wedge resection of

the attached left upper lobe, and wedge resection from the lingula to resect a solitary, possibly metastatic, coin lesion

Resection of the lower part of the sternum, the xiphoid process, and the left costal arch en bloc with a lung parenchyma wedge

Resection of costal cartilages and anterolateral parts of the second to sixth ribs on the left en bloc with part of the sternum, the left

pectoralis major and underlying skin, wedge resection of the left upper lobe and parietal pleurectomy from the apex to the fifth rib

Mesh reconstruction with dual-sided $2 \mathrm{~mm}$ e-PTFE mesh

Resection of lateral or posterior segments of $>4$ ribs

5

I

I

I

I

Resection of lateral or posterior segments of $>4$ ribs and lung wedge resections to remove the attached to the tumor lung parenchyma 2

Resection of the posterolateral parts of the fifth to eleventh ribs en bloc with the adjacent muscles, part of the left hemidiaphragm । and spleen

En bloc resection of the lower sternum, xiphoid process, right costal arch, and the upper part of the rectus abdominis musles

Abbreviation: e-PTFE, polytetrafluoroethylene.

Full-thickness chest wall resection was necessary in seven cases. Plastic surgeons were involved to cover the skeletal prosthetic reconstruction material in these seven cases. Latissimus dorsi musculocutaneous flaps were used in four cases, the combination of latissimus dorsi and pectoralis major muscular flaps was used in one case, and pectoralis major muscular flaps were used in two cases.

\section{Short-term outcomes}

Mortality of the procedures was nil. All patients were extubated within the first 24 hours in the intensive care unit (ICU). Postoperative ICU stay was $<48$ hours in all patients. Major morbidity occurred in four out of the 20 patients (20\%), comprising atelectasis requiring bronchoscopy and noninvasive ventilation for resolution in two patients, skin necrosis requiring initial vacuum-assisted closure application and an additional procedure from the plastic surgeons, and ischemia due to venous congestion of a musculocutaneous flap in one case requiring an additional procedure from the plastic surgeons in a second time.

Minimal microscopic invasion in the margin of the specimen was found at histologic examination in one patient (microscopic residual disease/R1 procedure). The patient underwent further completion of the chest wall resection and revision of the chest wall reconstruction, 3 weeks after the initial procedure, in order to achieve a final R0 oncologic result. The patient is free of any recurrence at 5 years of follow-up.
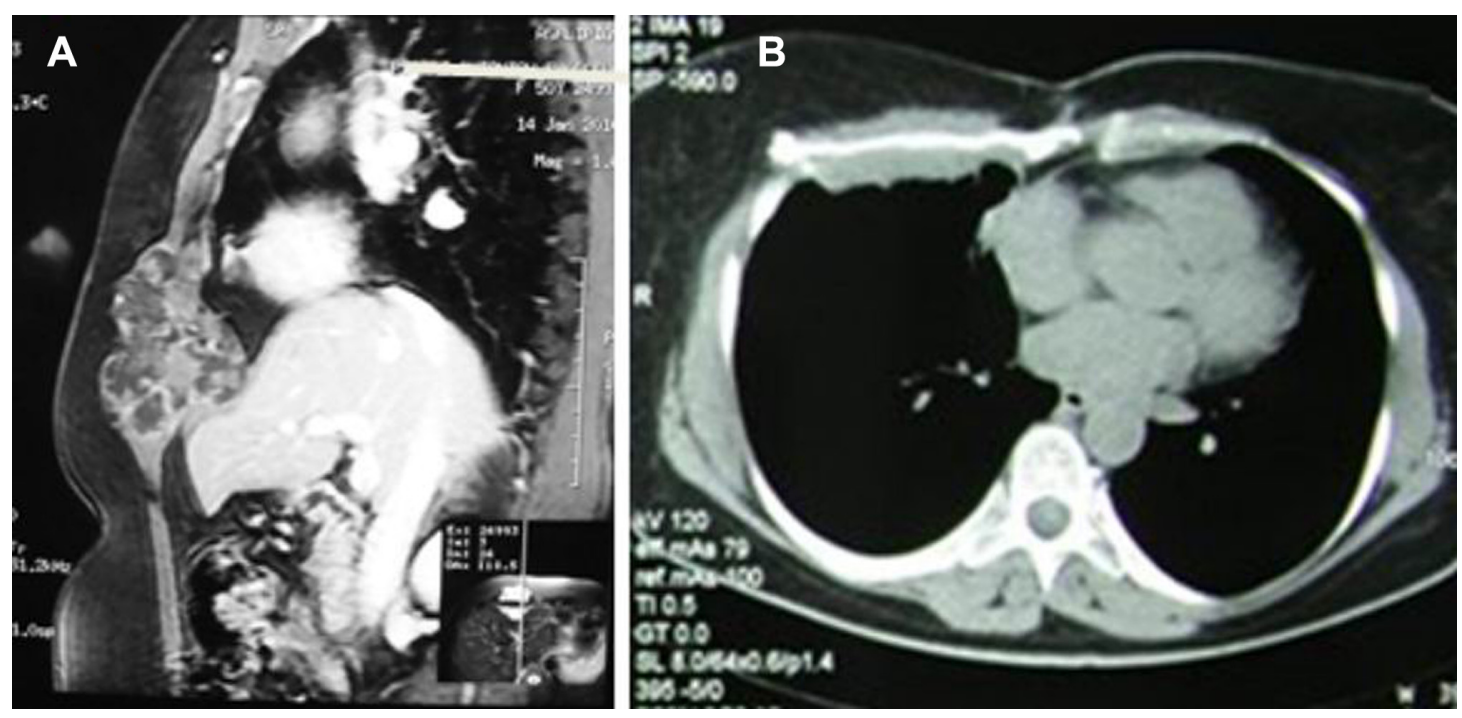

Figure 3 CT scans of the patient.

Notes: (A) CT scan reconstruction showing a chondrosarcoma of the right costal arch. (B) Reconstruction of the chest wall defect with e-PTFE mesh.

Abbreviations: CT, computed tomography; e-PTFE, polytetrafluoroethylene. 


\section{Adjuvant treatment}

Adjuvant oncologic treatment was offered in 13 patients. The patients with primary chest wall lymphoma underwent eight cycles of chemotherapy with the combination of doxorubicin, cyclophosphamide, vincristine, prednizolone, and rifuximab. Adjuvant treatment was not offered 1) in two elderly patients with associated cardiac problems, local recurrence occurred in one of them and 2) in five patients with low-grade chondrosarcomas and radical (R0) resection with wide-free margins. All the latter patients did not develop tumor recurrence.

\section{Recurrences and long-term outcomes}

Tumor recurrences were observed in five patients $(25 \%)$. All recurrences were local, while one patient developed beyond local recurrence and a solitary lung metastasis later on the course.

\section{Recurrent tumor \#I}

In this patient, local recurrence of a giant $(33 \times 26 \mathrm{~cm}) \mathrm{MFH}$, 6 months after initial resection and chest wall replacement with e-PTFE mesh followed by radiotherapy, was observed (Figure 4A). The patient underwent resection of his large recurrent tumor $(15 \times 13 \mathrm{~cm})$. The patient died 6 months after resection of the recurrence by developing one or more recurrence in the chest wall, close to the spine. The overall survival reached 12 months. Adjuvant radiotherapy did not prevent tumor recurrence, while chemotherapy was not offered due to the existing low left ventricle ejection fraction.

\section{Recurrent tumor \#2}

Here, an initial incomplete resection of the tumor in patient with MFH was done by nonthoracic surgeons. Massive chest wall resection and reconstruction were followed by local recurrence of the tumor just behind the methyl methacrylate prosthesis 8 months after the chest wall resection. The patient died 3 months later from the progressive disease not responding to the oncologic treatment, thus eliminating overall survival to 11 months. Adjuvant oncologic treatment did not prevent tumor recurrence.

\section{Recurrent tumor \#3}

In this patient, local recurrence of a large $(15 \times 12 \mathrm{~cm}) \mathrm{MFH}$ just above the mesh, 12 months after the resection of multiple ribs and e-PTFE mesh reconstruction, was observed. Local recurrence became evident as recurrent fluid collection with positive cytology above the mesh, without mass formation. The patient underwent resection of the soft tissues underlying the mesh, cover of the prosthesis with local flaps and radiotherapy. The patient is free of macroscopic disease and symptoms for 14 months after the completion of radiotherapy (overall survival until now of 26 months). The tumor was initially misdiagnosed and treated as subcutaneous abscess by a primary care nonthoracic surgeon.

\section{Recurrent tumor \#4}

In this patient, local recurrence of chondrosarcoma within the soft tissue in front of the prosthesis, 1.5 years after subtotal sternal resection and reconstruction with the sandwich technique and adjuvant chemoradiotherapy, was observed. Resection of the recurrence was made without the revision of prosthesis. The patient developed 2.5 years later a solitary metastasis in the superior segment of the left lower lobe that was treated with typical segmentectomy of the superior segment of the left lower lobe. He is now free of disease for 5 years since the
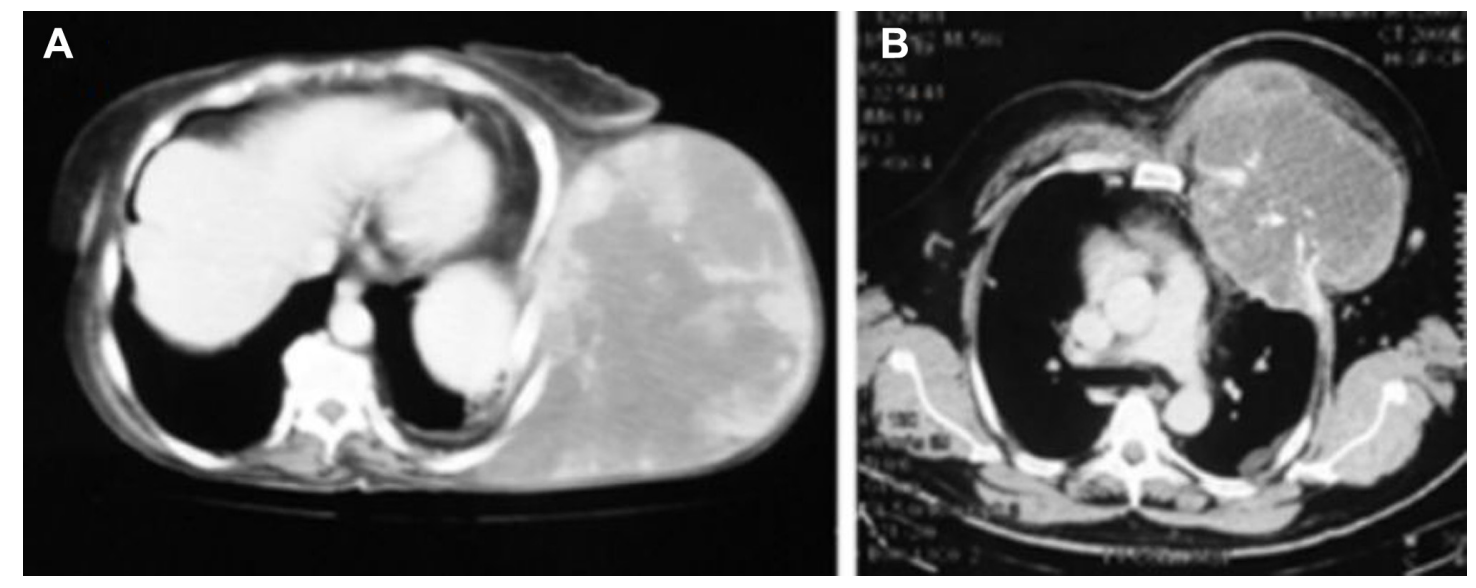

Figure 4 CT scan slices showing two large chest wall tumors where a local recurrence was observed after resection with tumor-free margins.

Notes: (A) Giant malignant fibrous histiocytoma of the chest wall measuring $32 \mathrm{~cm}$ in its maximal diameter. (B) Large chondrosarcoma measuring $20 \mathrm{~cm}$ in its maximal diameter.

Abbreviation: $\mathrm{CT}$, computed tomography. 
resection of lung metastasis (overall survival of 9 years). The patient underwent an initial incomplete resection of the tumor by a nonthoracic surgeon. Adjuvant chemoradiotherapy did not prevent the development of local recurrence.

\section{Recurrent tumor \#5}

In this patient, recurrence of chondrosarcoma within the pleural cavity at 7 months since the initial chest wall resection and reconstruction was observed. The patient underwent initially the resection of a giant chondrosarcoma $(20 \times 17 \mathrm{~cm})$ and parietal pleurectomy for pleural deposits in the apex of the hemithorax ( $\mathrm{R} 1$ resection). The patient died 5 months later due to the cardiac complications of chemotherapy with pazopanid. The overall survival was 12 months (Figure 4B).

All the five local recurrences occurred within a short period of time (6-18 months) after resection of the tumor. Recurrences were observed in two cases of giant tumors with maximum tumor diameters of $32 \mathrm{~cm}$ and $20 \mathrm{~cm}$, respectively (Figure 4A and B) and in three additional cases of initial incomplete resections or misdiagnosis as chest wall abscess that were performed by nonthoracic surgeons. The median survival of patients with recurrent tumors was 18 months; however, median survival was influenced by the prolonged survival of 9 years in the first of the recurrent tumors mentioned earlier. Survival was limited to 1 year in three out of the five patients with recurrent chest wall tumors. The three cases of MFH recurred locally within a short time period after resection (6 months, 8 months, and 12 months, respectively).

The rest 15 patients still remain alive without local recurrence or metastases during the whole follow-up period, and the median survival was 62.5 months. All the five patients with radically resected grades I and II chondrosarcomas still remain alive after follow-up of 20-108 months with a median survival of 62 months. The patients with primary chest wall lymphoma and with localized malignant mesothelioma are free of local or any other recurrence 4 years and 6 years, respectively, after the chest wall resection.

\section{Long-term consequences of massive chest wall resection and reconstruction}

Fracture of the sandwiched methyl methacrylate occurred once in 1.5 years after the resection of sternal chondrosarcoma (Figure 5). Fracture of the methyl methacrylate was incidentally discovered during the follow-up in chest CT scan. Fracture did not affect the stability of the prosthesis and chest wall, and the prosthesis was left in place.

Severe limitations of daily activities were observed in only one patient. The observed malfunction comprised the

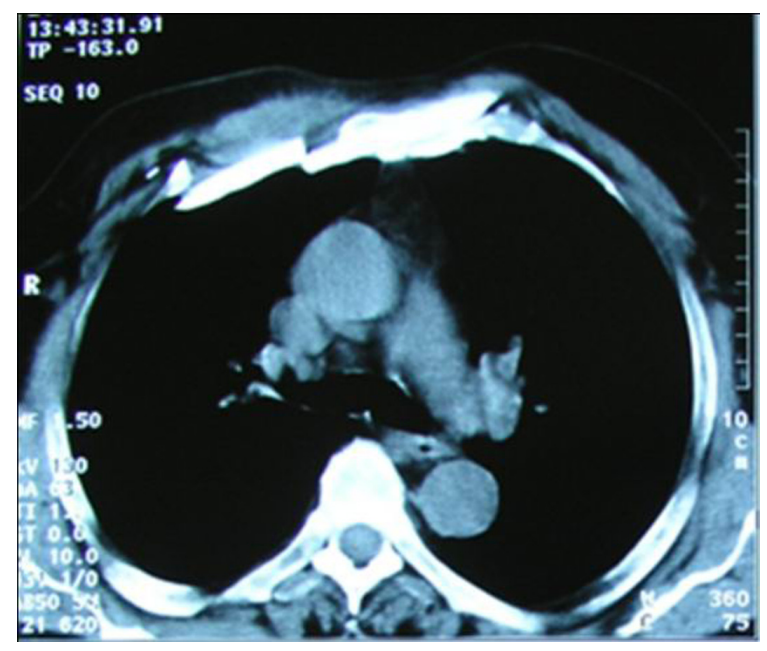

Figure 5 Asymptomatic fracture of the sandwiched methyl methacrylate prosthesis; incidental finding during routine follow-up CT scan performed at 1.5 years after resection of sternal chondrosarcoma.

Abbreviation: CT, computed tomography.

restriction in adduction and internal rotation of both upper arms, and it was the consequence of the resection of the upper part of the sternum en bloc with the attached head and anterior part of the clavicles for chondrosarcoma (Figure 6). Initial management by nonthoracic surgeons was observed in three out of the five recurred cases in the current series (recurrent tumor \#2, recurrent tumor \#3, and recurrent tumor \#4).

\section{Discussion}

Chest wall resection for tumor is well known since the late 19th century, as Holden was the first to describe partial sternectomy for a primary sarcoma in 1878 , followed by the report of chest wall resection for tumor by Parham in $1898 .{ }^{7}$ Moreover, highly successful chest wall resection and reconstruction with synthetic materials were applied since the early 1960s in Memorial Sloan-Kettering Cancer Center in New-York, and a case series of 155 patients was published in $1980 .^{8}$

Massive chest wall resection is currently performed for primary chest wall soft tissue, bony and cartilaginous primary chest wall tumors, and non-small-cell lung cancer invading the chest wall. Breast cancer infiltrating the chest wall (seen for the first time or locally recurrent) is no longer the case requiring chest wall resection. Surgery for lung cancer invading the chest wall involves additional major lung parenchyma resection en bloc with the involved part of chest wall, a situation that is quite different from primary or even metastatic chest wall tumors requiring chest wall resection. For the reason mentioned earlier, patients who underwent massive chest wall resection for lung cancer with chest wall invasion were not included in the study. 

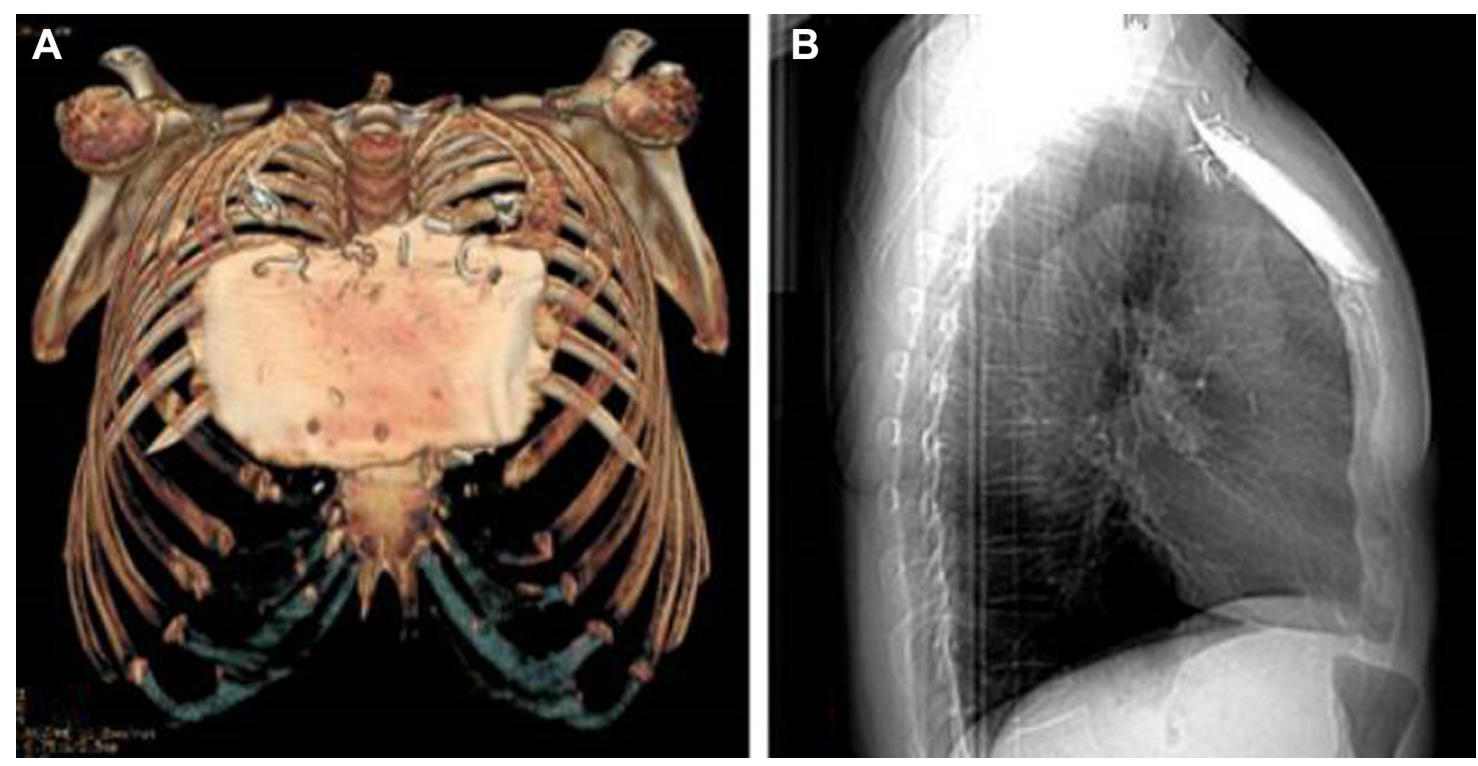

Figure $63 \mathrm{D}$ reconstruction of the sandwiched methyl methacrylate prosthesis in the patient experiencing restriction in adduction and internal rotation of both upper arms after resection of the upper half of the sternum.

Note: 3D reconstruction $(\mathbf{A})$ and; Chest $\mathrm{x}$-ray $(\mathbf{B})$. Fixation of the clavicles to the large sandwiched methyl methacrylate prosthesis with stainless steel sutures could be one of the responsible factors for patient's malfunction.

Abbreviation: 3D, three dimensional.

Chest wall reconstruction in this study has been made by using a certain strategy, the classic sandwich technique for anterior chest wall defects including the sternum or the dualsided e-PTFE mesh reconstruction for chest wall resections involving multiple ribs or the costal arch. The sandwich technique is well known from the early 1960s, and this method of reconstruction is associated with very good functional results concerning chest wall stability and upper arm and chest mobility. ${ }^{8}$ This technique was considered to be the best choice for reconstruction in case of total sternectomy in the near past. ${ }^{2,4,5,9}$ Encapsulation of the sandwiched methyl methacrylate prosthesis within a short period of time makes the construction permanently stable and resistant to the externally applied forces during daily life. Because of the encapsulation process, the methyl methacrylate prosthesis remains stable even in case of fracture of the sandwiched bony cement, as it was observed once in the current case series. "Loosening" and dislocation of the prosthesis requiring reoperation "due to ruptured sutures between the prosthesis and ribs" were reported by Lardinois et al. ${ }^{10}$ Fixation of the prosthesis with four stainless-steel sutures (one in each corner) to the surrounding bones according to the proposed herein technique ameliorates this possibility. Mesh reconstruction of the lateral chest wall has the disadvantage of flattening of the reconstructed area by the under tension mesh, which theoretically could alter the mechanics of ventilation by producing a restrictive pattern. Indeed, neither reconstruction of chest wall with sandwiched methyl methacrylate nor mesh was found to be associated with an important impairment of the pulmonary function. ${ }^{10,11}$ Fracture of the prosthesis is reported for the first time in our case series. Stability of the prosthesis/ chest wall due to the encapsulation process is an important finding of our work.

Other techniques and materials in use to cover the chest wall defects are the polypropylene mesh, the sandwich between two layers of polypropylene mesh stainless steel mesh or titanium plate or the titanium mesh itself. ${ }^{12-14}$ Recently, the titanium rib bridge system that bridges the ribs in combination with the dual-sided e-PTFE mesh, which forms the underlay to separate the thoracic cavity from the soft tissues of chest wall, has been used for chest wall reconstruction following oncologic chest wall resections by some institutions in Europe. ${ }^{15,16}$ Titanium bars in combination with sternal allograft are also used for chest wall defect reconstruction. ${ }^{17}$ The "rib-like" technique proposed by Girotti et al ${ }^{18}$ which combines a polyester-knitted mesh as underlay, and the combination of a radiopaque acrylic resin and methyl methacrylate resin to form the sternal substitute is another proposed technique for sternal reconstruction. No consensus, however, exists on which is the preferable material and the optimal technique to cover the large chest wall defects after chest wall resection, and the decision still remains the surgeon's choice. ${ }^{4,19}$ The e-PTFE mesh and the titaniummade prostheses/systems are quite expensive. In contrary, 
the classic and well-tested sandwich technique throughout the years still remains a valuable technique of minimal cost. The cost of a surgical technique is a very important parameter during the period of global financial crisis, which led the governments to make cuts in the available budgets for public health in most of the European countries. ${ }^{20}$

Size of tumor, tumor histology (worse prognosis for MFHs and favorable for chondrosarcomas), and initial incomplete resection of tumor made by nonthoracic surgeons were identified in the current case series as the main predisposing factors to recurrence. Adjuvant oncologic therapy was not able to prevent early recurrences in these five cases, while all the five recurrences were observed during the first 18 months after surgery. Recurrence rate of soft tissue tumors of the chest wall is higher during the first year after surgery, and thereafter, it declines each year according to McMillan et al. ${ }^{21}$ Tumor size at presentation was found to be an important predisposing factor in the series published by Walsh et al. ${ }^{3}$ Larger tumors cannot usually be resected with wide-free margins, and in addition, there is high possibility of spilling the operative field with neoplastic cells during the maneuvers made during resection. Concerning initial incomplete resections, the available data are not in agreement; ${ }^{22}$ Girotti et $\mathrm{al}^{18}$ support that initial incomplete resection is associated with worse prognosis, while Walsh et $\mathrm{al}^{3}$ did not find tumor recurrences after previous surgical curative attempts to affect prognosis if a complete resection can be achieved during the second procedure.

Recurrence rate and survival of chest wall tumors are strongly related to the histology of the tumor. ${ }^{13}$ Undifferentiated pleomorphic sarcoma (the alternate name advocated by World Health Organization to replace the term MFH in 2002) was in the series published by Chapelier et al and McMillan et al the commonest soft tissue sarcoma located in the chest wall, finding that is in agreement with the findings of the current series. ${ }^{5,22}$ MFHs of the chest wall tend to reach large dimensions and to recur after resection, even if macroscopic tumor-free margins can be achieved. Histologic grade of chest wall sarcomas was another predictor of survival in the series published by Incarbone et $\mathrm{al}^{2}$ and by Chapelier et al. ${ }^{5}$

Chondrosarcomas are the more common primary malignant chest wall tumors, accounting also for the $43.7 \%$ of primary chest wall sarcomas in our case series. ${ }^{13,14,23,24}$ Recurrence rate of chondrosarcomas is very low if radical resection with wide tumor-free margins can be achieved. ${ }^{2,3,9}$ The recurrence rate of chest wall chondrosarcoma was found to be high $(33 \%)$ in a series of 96 chondrosarcomas published in 2009 by the Scandinavian sarcoma group. Interestingly, the mean time to recurrence was 3.5 years after resection in this series. ${ }^{24}$ However, the large proportion of the included in the study patients had marginal $(45 \%)$ or incomplete (27\%) resections, the fact that is responsible for the high recurrence rate of chondrosarcoma in this series. In the study mentioned earlier, incomplete $\mathrm{R} 1$ and $\mathrm{R} 2$ resections and grade of tumor and treatment offered in a specialized sarcoma center seriously affected the survival. On the other hand, the authors did not find any correlation between the size of tumor and recurrence. In contrary to the Scandinavian sarcoma group, Burt did not find any difference in survival between the three grades of chondosarcoma in their series of 25 chondrosarcoma cases. Surprisingly, the Scandinavian sarcoma group point out that centralization of treatment of chest wall chondrosarcoma in sarcoma centers is more important for a better outcome than performance of surgery by thoracic surgeons or orthopedic oncologists. ${ }^{24}$ The pattern or first recurrence (local or distant) was found to be another factor affecting the survival in chondrosarcomas in the series published by Burt et $\mathrm{al}^{1}$ and Burt. ${ }^{23}$

Concerning the other tumor histologies, resection is the best way to achieve the local control in primary chest wall Ewing's sarcoma according to the results of the EURO-EWING 99 trial, making radiotherapy unnecessary if complete resection can be achieved. ${ }^{25}$ Moreover, primary chest wall non-Hodgkin lymphomas presenting as solitary chest wall masses are quite uncommon, and they represent $<2 \%$ of all chest wall tumors. Radical surgery followed by adjuvant chemotherapy was reported in small series and case reports to provide satisfactory local control and survival in patients in whom the chest wall was the only site of disease. ${ }^{26,27}$ In the details reported here, case complete resection of the mass together with the deposit in the spleen is associated with recurrence-free 4-year survival.

Respiratory or any other functional compromise was not observed in the patients of the study, with the exception of one patient who experienced restriction in adduction and internal rotation of both upper arms. Fixation of the remnants of both clavicles to the large methyl methacrylate prosthesis could be a possible explanation. Overall, the functional result of the reconstruction of large skeletal chest wall defects was very good in our case series of 20 patients, finding that is in total agreement with the excellent physiologic results reported in the series published by Lardinois et al, ${ }^{10}$ by Mansour et al, ${ }^{4}$ and recently by Leuzzi et al. ${ }^{28}$

The relatively small number of patients, the heterogeneity of the included chest wall tumors, and the variance in adjuvant treatment are the most important limitations of this 
study, which is a single-institution case series. On the other hand, chest wall reconstruction was made with the same strategy, and the applied techniques - mesh or sandwiched methyl methacrylate reconstructions - are well established and reproduced with the same way. The lack of variation in the surgical technique renders the results valuable. The major remarks that can be drawn from the study are as follows: 1) Stabilization of the sandwiched methyl methacrylate by some stainless steel sutures to the surrounding bones ameliorates the complication of prosthesis dislocation. 2) Fracture of a chest wall sandwiched methyl methacrylate prosthesis do not require revision of the reconstruction because it is very stable due to the encapsulation process. 3) Reoperation in case of $\mathrm{R} 1$ resection to obtain a final $\mathrm{R} 0$ resection should be made because it gives the patient the best chance for prolonged survival.

\section{Conclusion}

In conclusion, extensive chest wall resection and reconstruction for chest wall malignancies are technically demanding surgery. Primary chest wall tumors are rare mesenchymal neoplasms, which require major chest wall resection for their eradication. Favorable prognosis for most of the chest wall tumors is expected if complete resection with wide tumorfree margins can be achieved, because massive chest wall resection followed by reconstruction is feasible with low complication rate. Plastic surgeons should be involved in the surgical team to cover the prosthetic materials with viable tissues in cases where full-thickness chest wall resection is expected. Poor survival after massive chest wall resection for chest wall tumors is related to the large size of tumor, tumor's histology, as MFHs tend to recur, and inappropriate initial management by nonthoracic surgeons, especially macroscopic incomplete resections.

\section{Acknowledgment}

The findings of this article have been presented as an oral presentation at the 29th European Association for CardioThoracic Surgery (EACTS) Annual Meeting, Amsterdam, the Netherlands, October 3-7, 2015, by Dr Christoforos Foroulis.

\section{Disclosure}

The authors report no conflicts of interest in this work.

\section{References}

1. Burt M, Fulton M, Wessner-Dunlap S, et al. Primary bony and cartilaginous sarcomas of chest wall: results of therapy. Ann Thorac Surg. 1992;54(2):226-232.
2. Incarbone M, Nava M, Lequaglie C, Ravasi G, Pastorino U. Sternal resection for primary or secondary tumors. J Thorac Cardiovasc Surg. 1997;114(1):93-99.

3. Walsh GL, Davis BM, Swisher SG, et al. A single-institutional, multidisciplinary approach to primary sarcomas involving the chest wall requiring full-thickness resections. J Thorac Cardiovasc Surg. 2001;121(1): $48-60$.

4. Mansour KA, Thourani VH, Losken A, et al. Chest wall resections and reconstruction: a 25-year experience. Ann Thorac Surg. 2002;73(6): 1720-1725; discussion 1725-1726.

5. Chapelier AR, Missana MC, Couturaud B, etal. Sternal resection and reconstruction for primary malignant tumors. Ann Thorac Surg. 2004;77(3): 1001-1006; discussion 1006-1007.

6. Lin GQ, Li YQ, Huang LJ, Luo FY, Jiang HH, Luo WJ. Chest wall tumors: diagnosis, treatment and reconstruction. Exp Ther Med. 2015;9(5): $1807-1812$.

7. Sala L. [Sarcoma of the sternum]. Arch Sci Med. 1958;105(4):404-409.

8. McCormack P, Bains MS, Beattie EJ Jr, Martini N. New trends in skeletal reconstruction after resection of chest wall tumors. Ann Thorac Surg. 1981;31(1):45-52.

9. David EA, Marshall MB. Review of chest wall tumors: a diagnostic, therapeutic, and reconstructive challenge. Semin Plastic Surg. 2011;25(1): $16-24$.

10. Lardinois D, Muller M, Furrer M, et al. Functional assessment of chest wall integrity after methylmethacrylate reconstruction. The Annals of thoracic surgery. 2000;69(3):919-923.

11. Jonsson P, Gyllstedt E, Hambraeus G, Lillogil R, Rydholm A. Chest wall sarcoma: outcome in 22 patients after resection requiring thoracic cage reconstruction. Sarcoma. 1998;2(3-4):143-147.

12. Haraguchi S, Hioki M, Hisayoshi T, et al. Resection of sternal tumors and reconstruction of the thorax: a review of 15 patients. Surgery today. 2006;36(3):225-229.

13. Chapelier A, Macchiarini $\mathrm{P}$, Rietjens $\mathrm{M}$, et al. Chest wall reconstruction following resection of large primary malignant tumors. European journal of cardio-thoracic surgery: official journal of the European Association for Cardio-thoracic Surgery. 1994;8(7):351-356; discussion 357.

14. Yang H, Tantai J, Zhao H. Clinical experience with titanium mesh in reconstruction of massive chest wall defects following oncological resection. Journal of thoracic disease. 2015;7(7):1227-1234.

15. Fabre D, El Batti S, Singhal S, et al. A paradigm shift for sternal reconstruction using a novel titanium rib bridge system following oncological resections. European journal of cardio-thoracic surgery: official journal of the European Association for Cardio-thoracic Surgery. 2012;42(6):965-970.

16. Berthet JP, Canaud L, D'Annoville T, Alric P, Marty-Ane CH. Titanium plates and Dualmesh: a modern combination for reconstructing very large chest wall defects. The Annals of thoracic surgery. 2011; 91(6):1709-1716.

17. Dell'Amore A, Cassanelli N, Dolci G, Stella F. An alternative technique for anterior chest wall reconstruction: the sternal allograft transplantation. Interactive cardiovascular and thoracic surgery. 2012; 15(6):944-947.

18. Girotti P, Leo F, Bravi F, et al. The "rib-like" technique for surgical treatment of sternal tumors: lessons learned from 101 consecutive cases. The Annals of thoracic surgery. 2011;92(4):1208-1215; discussion 1215-1206.

19. Deschamps C, Tirnaksiz BM, Darbandi R, et al. Early and long-term results of prosthetic chest wall reconstruction. The Journal of thoracic and cardiovascular surgery. 1999;117(3):588-591; discussion 591-582.

20. Quaglio G, Karapiperis T, Van Woensel L, Arnold E, McDaid D. Austerity and health in Europe. Health policy. Nov 2013;113(1-2): 13-19.

21. McMillan RR, Sima CS, Moraco NH, Rusch VW, Huang J. Recurrence patterns after resection of soft tissue sarcomas of the chest wall. The Annals of thoracic surgery. 2013;96(4):1223-1228. 
22. Jo VY, Fletcher CD. WHO classification of soft tissue tumours: an update based on the 2013 (4th) edition. Pathology. 2014;46(2):95-104.

23. Burt M. Primary malignant tumors of the chest wall. The Memorial Sloan-Kettering Cancer Center experience. Chest surgery clinics of North America. 1994;4(1):137-154.

24. Widhe B, Bauer HC, Scandinavian Sarcoma G. Surgical treatment is decisive for outcome in chondrosarcoma of the chest wall: a populationbased Scandinavian Sarcoma Group study of 106 patients. The Journal of thoracic and cardiovascular surgery. 2009;137(3):610-614.

25. Bedetti B, Wiebe K, Ranft A, et al. Local control in Ewing sarcoma of the chest wall: results of the EURO-EWING 99 trial. Annals of surgical oncology. 2015;22(9):2853-2859.
26. Hsu PK, Hsu HS, Li AF, et al. Non-Hodgkin's lymphoma presenting as a large chest wall mass. The Annals of thoracic surgery. 2006;81(4): 1214-1218.

27. Qiu X, Liu Y, Qiao Y, et al. Primary diffuse large B-cell lymphoma of the chest wall: a case report. World journal of surgical oncology. 2014;12:104.

28. Leuzzi G, Nachira D, Cesario A, et al. Chest wall tumors and prosthetic reconstruction: A comparative analysis on functional outcome. Thoracic cancer. 2015;6(3):247-254.

\section{Publish your work in this journal}

OncoTargets and Therapy is an international, peer-reviewed, open access journal focusing on the pathological basis of all cancers, potential targets for therapy and treatment protocols employed to improve the management of cancer patients. The journal also focuses on the impact of management programs and new therapeutic agents and protocols on

\section{Dovepress}

patient perspectives such as quality of life, adherence and satisfaction. The manuscript management system is completely online and includes a very quick and fair peer-review system, which is all easy to use. Visit http://www.dovepress.com/testimonials.php to read real quotes from published authors. 